\title{
Exploration of Arabic reading, in terms of the vocalization of the text form by registering the eyes movements of pupils
}

\author{
Djemaia Bensoltana, Boualem Asselah \\ Cognitive Neuroscience and Behavioral, LBO/Faculty of Biological Sciences, University of Sciences and Technology Houari \\ Boumediene (USTHB), Algiers, Algeria \\ Email: d.bensoltana@gmail.com
}

Received 4 August 2013; revised 6 September 2013; accepted 16 September 2013

Copyright (C) 2013 Djemaia Bensoltana, Boualem Asselah. This is an open access article distributed under the Creative Commons Attribution License, which permits unrestricted use, distribution, and reproduction in any medium, provided the original work is properly cited.

\begin{abstract}
The intrinsic factors such as the visual constraints, extrinsic such as biomechanics, interface such as the support: the writing, the linguistics, the typography, the sense of progress of the language, have their impact on the habit, and the fittest and particularly the efficiency in reading. We suspect that circuits and zones involved in horizontal orientation "right to the left" are not the same the other way around hanging the linguistic collection. Reading Arabic provided with a specific difference in vocalization proves to be a valuable model verification two major issues which arouse language perception: [lexical processing: is it instantaneous (visual memory) or delayed (memory-tampon)? ...]. And a comparison with other models of language detection mechanisms and brain areas mnemonics which is involved in making information. Results are significatively: children vocalized mode is best seen. It must extend the duration of the mode without vowels. In our opinion, our study is original, and we introduced what a valuable tool in the diagnosis of visual and cognitive strategy by the biotechnology technical based on variations in terms of reading. This opens a horizon of opportunity for the application of the method to the Arabic language to determine the differences in eye size reflecting the effects of task variables in reading and a comparative study of other languages.
\end{abstract}

Keywords: Eye Movements in Reading-Learning in School; Method Photoelectric; Saccadic Ocular; Lexical; Vocalization in Arabic

\section{INTRODUCTION}

The shapes of the letters play an important role in cap- turing written information, and it belongs to the aspects of "contextual form", which have great impact on adoption of written norms (intonation and diacritical). Understating the sentence, in our view, depends on how far the reader masters grammar and context of the language if text is not accentuated. If the text is accentuated, particularly in case of novice readers, readings become easy to understand, while load increases the optical polarization. This form, which has become optical obstacles, and the experienced learners disposes the accentuation for his or her ability to understand its meaning, (as concluded in a previous study in our laboratory). Our aim is to develop an easy method in order to enable linguistic context in terms of vocalization of the texts by children and of Arabic Language novice learners in order to show the effect of different in the norms of accentuation in terms of optical and knowledge mechanism in order to get the inputs, which focus on concentration on values, in which the following hypothesis is developed. Would we extend the accentuation of the text to the years of study, or is this sufficient? The form depends on how far the learner has read the lexical contents and it is parallel to mastering language grammar; needs comprehensive training, and realization of defining the period of experimental trials to ensure the validity of the hypothesis.

\section{EXPERIMENTAL METHODOLOGY}

\subsection{Brief on the Methodology}

Thanks to the works of Javel [1], the vertical movement of the eyes is not only continuous but it has rhythmic jumps, which are gradually ordered. It may occur randomly when one is absent minded. It is programmed intention as it is in reading.

Successive studies were realized to define the process 
of reading [2], and perfect the techniques of recording to resolve the problems of analysis of the difficulties of reading on the legibility and on the eye motricity [3]. With the discovery of photoelectric under our study, processing technology have developed a great deal, which would allow us to know the time trends and dimensions of view of the reader, through following his eye movement system behavior [4], as well as keeping the experimental surrounding conditions, like what happens with normal cases.

\subsection{Process Steps}

The experiment is carried out in very calm classroom, in which each student sits on a chair, which we can easily recognize while sitting on a desk upon which the text is put on a holder. The student wears specific experimental glasses, installed on three photo-electric cells (Figure 1). This technology depends mostly on corneal reflection. The movement of eyes creates variation in rays break and reflects eye's responses behavior through pulses (Figure 2), which are interpreted into bio-electricity through the curves of the eyes. Such curves are in direct proportion with eyes movement, where fixed standard light is installed in the celling of the room and directed towards the text. The text has interesting stories in order not to make the reader feel bored while reading and to avoid the difficulty of coding, and to avoid studying more difficult text content (annex). The reader is required to start voiceless reading on the signal, without moving his or head. He or she has been informed that he will repeat the story in order to devote his concentration.

\subsection{Process Procedures}

Experimental procedure is applied on the mixed sample with regard to sex and level, and composed of subjects, who are practicing reading at the six grades, at Elementary school. The age of each is 12 years, as the study of

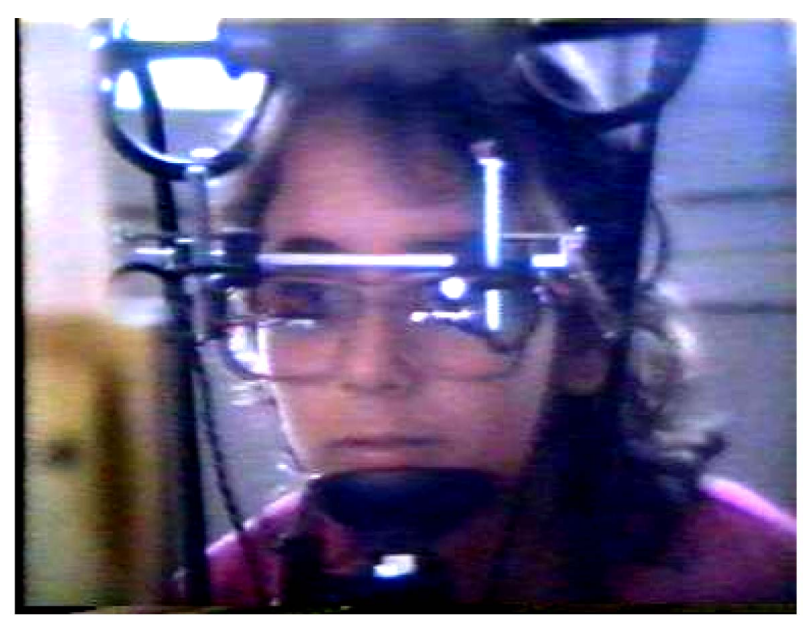

Figure 1. Pupil installed for reading by recording [5].

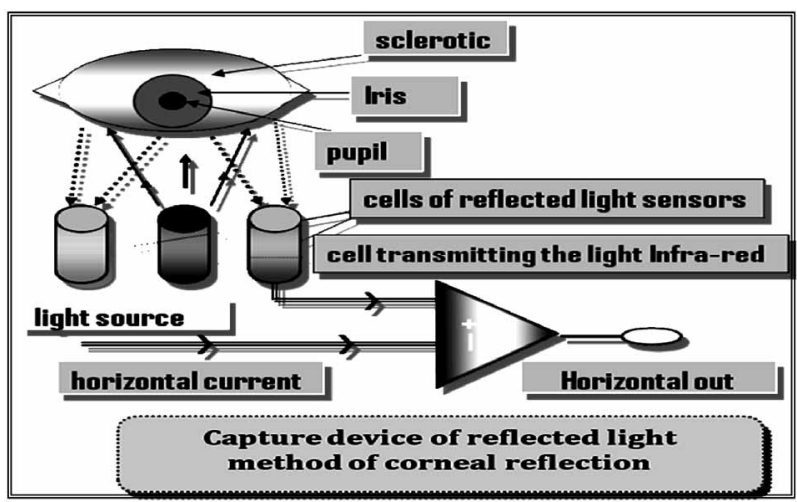

Figure 2. Technical photoelectrical for recording.

language grammar has been stabilized to some extent, at this age. The objective is to know the effect of how the text shape affects eyes movement, and to choose the appropriate method which responds to the vision of the student at this level.

Each student shall read recordings of three different texts, which differ in form (totally vocalised), and partially shape (ambiguous words), without accentuation and the effect on the sample is measured.

\subsection{Study of Eye Curves Recorded by Photo Electricity "Eye Curves"}

Study of transitional movements samples which were carried out through planned recordings. The horizontal movement for vision is recorded while reading, through survey for text from right to the left by successive pulses. The horizontal movement for vision is recorded gradually as grading steps (Figure 3). Then a record paper 25 $\mathrm{mm} / \mathrm{second}$ is to be withdrawn and the two figures are noted.

Standard aspects of the sizes of the abstracted rapid moves of eyes in the experiment are represented in terms of the following:

-Duration of fixation (DF), fixing the eyes on the words and coding this.

-Times necessary for reading (TR), it is the duration done each line in reading.

-The number of fixation (NF), represented in progressive grading in addition to recessive grading.

\section{RESULTS}

As per the following schedules and figures, the outputs of the experiment for each variable in inclusive (Table 1) and to represent comparison curves and to analyze them statistically.

A) The following (Table 1) combines the ocular parameters obtained function type of vocalization of the text.

B) The following results represent chart for three 


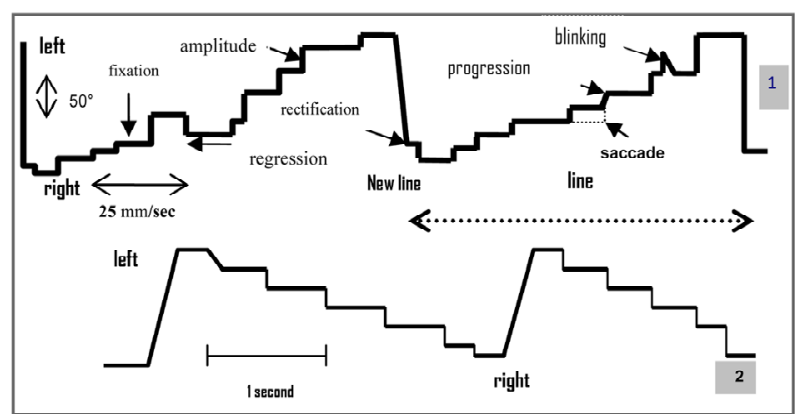

Figure 3. Two oculograms whose sense of reading is reversed [6]. Arabic $(\mathrm{R} \rightarrow \mathrm{L}) /$ French $(\mathrm{L} \leftarrow \mathrm{R})$.

Table 1. Variables resulting from the effect of vocalization form text on the eye movement.

\begin{tabular}{cccc}
\hline Aspect Variable & vocalic & Partially vocalic & No vocalic \\
\hline DF (msec/word) & 328 & 384 & 409 \\
TR (sec/line) & 4.81 & 5.97 & 6.22 \\
NF/line & 12 & 13 & 13 \\
\hline
\end{tabular}

curves, three for standard aspects as per the following form (Figures 4 (a)-(c)).

C) Analysis statistic: Results have shown the interlinked relationship between dependent variables which represent standard measurement for the independent sample, representing the form module (Table 2).

Interpretation: The results have shown that there is gradual decrease for the values as follows: (NV-PV-VC) for the 3 variables, mostly the third variable. There is brief about the vocalized form, as the preference value is available, then it is followed by the text in which the letters have been vowel and may lead to ambiguity, which hinders understanding of "partially accentuated". Then, it is followed by the text which is "total accentuated". Similarly, the aspects have taken the same trend in the statistical study.

\section{DISCUSSION}

Of the main problems facing reading Arabic language is the form of text itself, which forms great contradictory puzzle. Several questions and justifications have been raised in this field in order to find solutions for this problem but in vain. This may go back to economic reasons concerning typing or media, whether this information is "pedagogical, cultural, information or ideas", most information is not accentuated. In preliminary school level, the form of the text goes hand in hand with the norms of education, and studying grammatical system. This stage can be sufficient only with deleting the form through successive educational stages "Intermediate, Secondary, and University" levels.

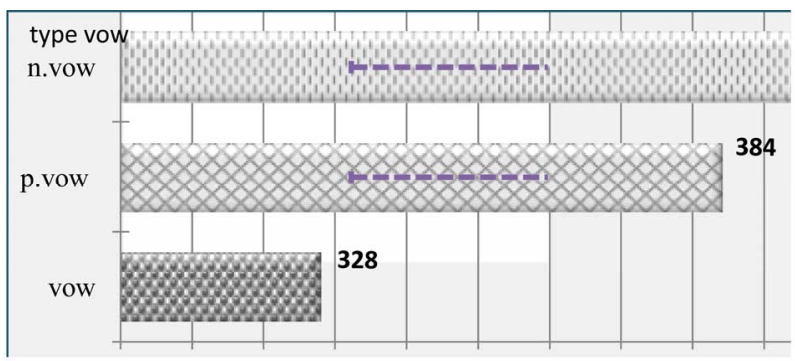

(a)

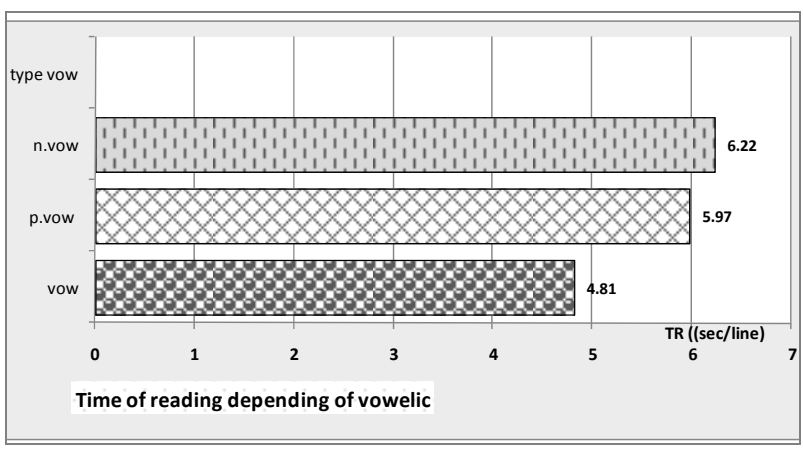

(b)

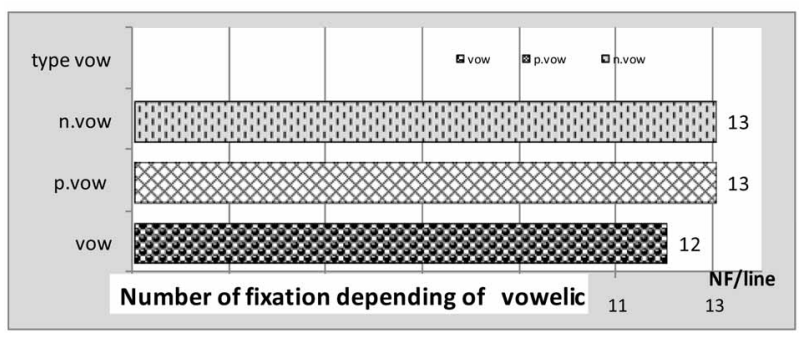

(c)

Figure 4. (a) representing the influence of three modes on the duration of fixation; (b) representing the influence of three modes on the duration of reading; (c) representing the influence of three modes on the number of reading.

If the words are accentuated, with full coding, understanding will be $95 \%$ correct. If the form is deleted, the reader hesitates in decision of getting the meaning and chooses superficial translation, for about $60 \%$ of sentences, for the final decision is not taken. On the other hand, the partially accentuated sentences are (passive voices) are read clearly also, but they are misrepresented to some extent if the text is not fully accentuated. "The vocalization is used to remove ambiguity in the lexicon during reading".

- On reading no-accentuated texts, the decision taking has been linked with the higher level since the long term memory, which is rooted in childhood. Hence reading non accentuated Arabic text requires recognition and knowledge effort to the top centers, in which groups of tools, "The eyes play secondary role for understanding precedes reading".

- Approximately, there were only no-accentuated texts, 
Table 2. The effects of the analysis of variance of two tests on parameters oculars.

\begin{tabular}{|c|c|c|c|c|c|c|c|c|}
\hline \multicolumn{2}{|c|}{ Variable test } & $\mathrm{DF} \int \mathrm{Fc}$ & $\mathrm{TL} \int \mathrm{Fc}$ & $\mathrm{NF} \int \mathrm{Fc}$ & Variable test & $\mathrm{DF} \int^{\circledR}$ & $\mathrm{TR} \int^{\circledR}$ & $\mathrm{NF} \int^{\circledR}$ \\
\hline \multicolumn{2}{|c|}{$\mathrm{Fc}$} & 6.37 & 7.65 & 1.50 & ${ }^{\circledR}$ & -0.98 & -0.94 & -0.90 \\
\hline \multirow{2}{*}{$F_{\text {théorique }}$} & $\mathrm{T}_{0.05}$ & 3.07 & 3.07 & 3.07 & pente & Negative & Negative & Negative \\
\hline & $\mathrm{T}_{0.01}$ & 4.79 & 4.79 & 4.79 & Conclusion & strong link & strong link & strong link \\
\hline \multicolumn{2}{|c|}{ Interpretation } & very s. & high s. & No s. & Test de fisher ( $\mathrm{F}$ & ed)-Coeffic & e correlatio & gnificative: $\mathrm{s}$ \\
\hline
\end{tabular}

which goes back to low effort law and cost of typing.

- In comparison between Arabic Language reading, particularly touches Latin Language, which is distinguished by knowledge recognition and is run by two functions:

Objective $\rightarrow$ Optics $\rightarrow$ Knowledge recognition.

Arabic language which intermediates "interlinked routes" is processing route within reading non accentuated text: method of integrating grammatical analysis or vowels, or integration, while reading is devoted in three routes:

Objective $\rightarrow$ Optics $\rightarrow$ Analytical analysis $\rightarrow$ Knowledge recognition.

Of main incentives for this study is what we noted during learning that the children, in comparison with children who study Latin, exert much effort to learn the linguistic context, which is not accentuated. That is because non accentuated text requires in depth mastering for language grammar. The student is to learn two plans:

- Adoption Module: in which optical mechanism is adopted on accentuated system and by the end of preliminary stage, the fixation of non-accentuated modules is planted in the head, after completion of linguistic explanation (grammar-transformation and morphology).

- Complementary Module: This module completes Final grade of preliminary school in which language inputs memorization fixing is replaced with removal of the accentuation. Whereas Latin primary stage learners make use of "Lack of explanatory efforts" exerted of optical knowledge as the form is integrated in the context of the word and reading it is provided in the incidental moment spend in understanding the text.

This study has leaded us to raise the following two questions.

Question (1): Does reading texts without vowels impose an extended period for learning which prevents it from progressing?

Question (2): Does reading in terms of form, after considering lines spaces, which are narrow, decrease the polarized load on eyes to facilitate understanding on one hand and hinder vision from another?

The function of form is to record the optical memory to which the reader resort to in order to understand quickly and when removed, he has to revert to the brain memory.

According to the results obtained from study on School children, the best performance is too related to reading accentuated texts, so we suggest in this regard, that indirect knowledge trends is adopted to express understanding for in learning "form style is very necessary to compensate the necessity for developing " Tran-center in which explanation is introduced". This means that words accentuation is included among basic physical properties needed to possess and facilitate the access to the better linguistic understanding; but we collected exaggerated results to some extent. This goes back into the increase of optical recognition load or increasing spaces between lines, which in its turn would compensate economic knowledge and does not bring a proactive explanation.

Through this conclusion, we can discuss two contradictory theories regarding in the two issues "A Comparison carried out by physic-psychological researchers on the movement of eyes" during reading [7] and whether the translation of optical information is instant or postponed in the brain [8]. We can explore that reading Latin is subject to instant norm, but in Arabic, reading is made with a view to get the following information:

In Education stage, writing imposes a problem in which the instant norm plays its role, that is the intervention of "Optical Activity", but in case the form is removed in next stage, knowledge recognition route is prepared, and the storage memory intervenes. Levy Schoen, [9], indicates that readers have the capacity to analyze sentences terms of information connected to the way its context is organized.

This gives explanation to-Extension of reading period-of Arabic text, which is not accentuated as a result of re explaining the word in order to understand a sentence that is there is delayed brain programming.

Reading Arabic texts is accused of having abbreviated stenographic, as it concerns only with necessary syntax for reading and accordingly it enforces the reader to use lost marks in hesitation to add programmed marks, as narrated by his resonance, but the marks of (vowels, dictation and grammatical symbols) are available!

[For it is not an error to write in the main time others refraining from using it $\cdots$ ?] 
We suppose the objective that studies carried out in this field were not built on scientific exploration, which match optical capabilities with efficiency.

If Arabic langue is different from Latin, this is not a weak point itself that is because it performs its function completely. The norm of linear criteria expresses the economic textual form during edition, which is explicit and simple and full [10]. But Arabic Language for economic technology increase thinking effort and depends on the long range memory and here the puzzle lies. In educational stages, and through the first levels, the problem does not come in the same manner as in next stages. The books are accentuated. The omission of letters is supported according to the norm of education "stability of grammatical rules". They build upon these stages to achieve this. This is the reason why the elementary stage has characterized by deleting the accentuations "form". This proves that the central nervous police is typical and programmed for reading which is accentuated in the form, and give more spaces for brain knowledge to which the reader resort to in reading non accentuated text. In our study, we shed light upon the different methods for setting the form in order to supervise sensual recognition and optical movement. We noted that eyes movement determines the best improvement for facilitating optical knowledge. We preferred this method to analyze reading process through evaluation of criteria, which have been detected by technology. It is the best method to be used to improve the text system from theoretical point of view [4].

\section{- Based on these results, we can conclude that:}

The form-accentuation marks facilitate reading and the access to Arabic lexicon. These are important signs to show the works for which direct circle for information translation is made without reference to knowledge system that is the lowest thought efforts, and economic period. Accordingly, repeated reverted circle of the decision is eliminated, and this applies on the instant theory mentioned above.

The main disadvantage of methodology as we mentioned before is the problem of accentuation form. The used signs have their significance and indicate that there is intervention which may lead to misunderstanding or load on eyes.

Arabic Language completes its function in an economic manner tailored according to adaptation with optical strategy and the methods to achieve this must be considered $[6,11]$.

Its repair requires more period-time patch. "If we accept the idea of integrating signs with consonants" as it was suggested by some researcher, since a century. Of course, this would be positively reflected upon novice learners."

\section{RELATED STUDIES}

Some studies have been made to face this problem in
Cairo Academy whose aim is to 'Establish new approach for form-accentuation elements necessary to put printing norm in more realistic manner; to find alternatives which simplify the principles of the experimental base, which has been defined in experimental method. Among other researchers for development projects are: Taimor (1951) from Egypt, Mohamed El Foraak (1952) from Syrian; in [12,13] from Morocco, [11]. Regardless the researches promoted by Cairo Academy, "Mesbakah" Western discoveries has provided solution for technical problem regarding textual form, for Monotype Printing House has adopted Accentuated Arabic writing printings. The solution proposed for temporal remedy for suffering which has been checked in bad manner. No modification was made upon this system. The system, for Lakhadr Ghazal, includes in addition to letters, signs, and punctuation marks, which can be overviewed in terms of criteria represented in modification, like Latin Language criteria. Accordingly, adoption with operation and machines manufactured on these criteria (economic method), the above mentioned projects did not succeed in finding solutions for shaping.

Assembly of printing experiments to correct existing problems is not accompanied by technical or behavioral experiments, to be checked under vocational supervision in optical mechanism or visuo-cognitive [14]. Our study can be subject to diagnostic or correctness of a decision, which aims to modifying form norm associated with education. We, through our study, join the researchers who aim to find correct solutions in order to ideologically improve and to meet the demands of the application and mechanism of optical recognition, knowledge and accentuated form repair [4].

\section{CONCLUSION}

Inputs of eyes behavior have proved that there is proactive response to accentuated text. Despite the fact that the sampled subjects are experienced to some extent in reading, yet it does not reach its maturity form by the end of preliminary study. In order to understand the text well, the shape shall be considered and extended for next years. Its removal depends on the studies defining experiments. So, it is preferred to extend it to the medium in order to be adopted with education and improve apagogical return. Our previous study which has been applied on the same experimental procedures on "students who study Arabic Language", whose age is about 22 years. The removal of shape does not require that the learners must be well trained on the use of Arabic language. Comparison of results concerning the period of fixing the word has shown that the result has proved to be reversible and contradicting. The response intends to improve the text without accentuation. Then it is moved to partial accen- 
tuation to total accentuation. At this stage, we have been able to overcome the inputs of language and imprinted in the stored memory for all linguistic interventions. The linguistic mastered learner prefers analysis of information in the direction of knowledge and thinking recognition [15]. We should not ignore the role of accentuation form in the contribution of information translation, and its effect on optical polarization recognition and to simplify it for understanding if adopted with the optical behavioral ability. This must apply on every novice learner (dialectic flexibility in Arabic language). Eventually, this study is included among psychological, linguistic and nervous studies, in which knowledge and recognition function supersedes optical psychology to explain the meaning of information [16]. Exploration of ideal style in how to make the text simple and in the meantime it goes back to economic reason such as printing (Book texts, editions, and journals, etc.), this would open new horizons for media: $[6,11,17,18]$. So that, in perspective, this would open this methodology for a wide prospect for improvement of the language, and carrying out the experiments, which touches processing linguistic contexts in all aspects associated with photo electricity of optical mechanism.

\section{REFERENCES}

[1] Javal, E. (1878) Original works: Try on the physiology of reading. Annals of Oculistiques, 82, 243-253.

[2] Levy-Schoen, A. (1969) The study of eye movements, rev. technical and knowledge. Collection Behavioral Science Book, Published in Conjunction with the National Center for Scientific Research. Dunod, Paris, 261.

[3] Baccino, T., Salmeron, L. and Cañas, J.J. (2007) Reading hypertext. In: Chevalier, A. and Tricot, A., Eds., Ergonomics of Electronic Documents, PUF, Paris.

[4] Mielletufr, S. (2004) Médiation phonologique, Accès lexical et contrôle oculomoteur en lecture. Thèse de Doctorat en Psychologie Unité de Recherche sur l'Evolution des Comportements et des Apprentissages universite, Lille III.

[5] Bensoltana, D. (2004) Mechanisms intervening in the elaboration of the driving plan of ocular movements saccadic during the reading in Arabic by Algerian child in primary school, LBPO, Neurosc. behav. and cognit., p153, no pub, FSB, USTHB, Algier.

[6] Bensoltana, D. and Asselah, B. (2005-2012) Research through the preparation of doctoral thesis, factors de- termining learning by recording eye movements in children while reading, behavioral and cognitive neuroscience, FSB, USTHB, Algeria.

[7] Just, M.A. and Carpenter, P.A. (1983) What your eyes do while your mind is reading. In: Rayner, K., Ed., Eye Movements in Reading: Perceptual and Language Processes, Academic, New York.

[8] Mc Conkie, G.W. and Yang, S.-N. (2004) How cognition affects eye movements during reading. In: Hyönä, J., Radach, R. and Deubel, H., Eds., The Mind's Eye: Cognitive and Applied Aspects of Eye Movement Research, Elsevier, Amsterdam, 413-427.

[9] Levy-Schoen, A. (1983) Measuring eye movements why? Group Regard, Psychol. Experimental, CNRS, Paris V., EPHE. Human Labor, 46, 3-9.

[10] Lakhdar, G.A. (1974) General methodology of Arabisation level. The system Lakhar-Ghazal, communication in the days of information between Arabic and French language, organized by the CILF, 1976. IERA, Grenoble, 29-30.

[11] Hamm, R. (1975) For the arabic typography. Technical Contribution to the Democratization of Arab Culture, Library Arabic, Paris, Syndbad, 193 p.

[12] Meynet, R. (1971) The Arabic script in question. Projects of the Academy of the Arabic Language in Cairo de 1938 à 1968, Beyrouth, Liban, 24 p.

[13] Lakhdar, G.A. (1967) About Arabic and the level of teaching Arabic. Lakhdar-Ghazal System Composition of Standard Arabic. Printing the REIA, Rabat, 1-25.

[14] Valdois, S. (2010)

[15] Spragins, A.B., Lefton, L.A. and Fisher, D.F. (1976) Eye movements during reading and Space Research transformed text: The development of the examination. Memory and Cognition, 4, 16-42.

[16] Calvo, M.G. and Meseguer, E. (2002) Eye movements and processing stages in reading: Relative contribution of visual, lexical, and contextual factors. The Spanish Journal of Psychology, 5, 66-77.

[17] Russel, G. (1988) The conditions of learning of reading, Adaptation strategies objectives reading: Learning of reading and the sign language. In Downing et Fijalkov (1990), Gwënola Paranthoën, 5-18.

[18] Belaïd, S. (1995) Domains and scientific subjects concerning designs responding to the century in the Arabic language. 